\title{
Evaluating competency based education modules in an online nurse practitioner course
}

\author{
Julie Worley, Michelle Heyland \\ College of Nursing, Rush University, Chicago, Illinois, United States
}

Received: September 12, 2018

Accepted: October 22, 2018

Online Published: November 8, 2018

DOI: $10.5430 /$ jnep.v9n3p1

URL: https://doi.org/10.5430/jnep.v9n3p1

\begin{abstract}
Competency based education (CBE) has been shown to improve academic performance and could help bridge the gap between education and clinical practice. There is a lack of evaluation data for new content added to courses, particularly CBEs and new technology. The aim of the study was to evaluate the use of CBE modules and GoReact technology in an online psychiatric nurse practitioner course. In a quality improvement study, four CBE modules were used to assess knowledge and clinical skills in an online psychiatric assessment course. Knowledge tests were used to assess student knowledge, adaptations of the Student Evaluation of Educational Quality Scale (SEEQ) and the Systems Usability Scale (SUS) were used to evaluate the students' responses to the CBE modules. Faculty feedback and comparisons from prior years without CBEs were also examined. All students in the course successfully completed the CBE modules for course credit. The majority of the students who completed the surveys had a positive response to the CBEs and GoReact technology. Faculty were satisfied with using CBEs and the technology and overall student performance in the course and subsequent practicum course following the CBEs was the same or improved. CBE modules appear to be an effective and well received method of instruction in online clinical psychiatric nurse practitioner courses.
\end{abstract}

Key Words: Competency based education, Advanced practice nurse, Psychiatric nurse practitioner, Teaching strategies, Evaluation, Online education, Technology

\section{INTRODUCTION}

\subsection{Introduction to the problem}

Competency based education (CBE) is a teaching strategy where modules are added to courses and students then complete them on a flexible basis, enabling them to show mastery of content at their own pace and make good use of technology. ${ }^{[1]} \mathrm{CBE}$ has received attention in the educational literature over the past 20 years and aims to develop students' potential abilities and focuses on outcomes of learning. ${ }^{[2]}$ It has been shown that CBE modules improve academic performance and could help bridge the gap between education and clinical practice. ${ }^{[3]}$ An education program can be entirely
CBE based, or CBE modules can be incorporated into a traditional educational program. In nursing education, there is a lack of research on the use of CBE education and its impact on national certification exam results. ${ }^{[4]}$ However, it is generally agreed that CBE modules will likely play a significant role in nursing education in the future. ${ }^{[5]}$ The outcome-based approach of CBE's focus on learners' performance and outcomes on specific objectives and curricular goals is ideal in nursing because nursing programs prepare students to master concepts and skills that they will be tested on in national licensure and certification exams. ${ }^{[6]}$

*Correspondence: Julie Worley; Email: julie_worley@rush.edu; Address: College of Nursing, Rush University, Chicago, Illinois, United States. 
There is a paucity of research in the literature about implementing and evaluating CBEs into courses, particularly in nursing. The purpose of this quality improvement study was to evaluate the introduction of CBEs into an online psychiatric nurse practitioner clinical management course using outcome measures.

\subsection{Literature review}

In a European study, researchers examined task performance and assessment skills in 90 undergraduate nursing students using CBE concepts. ${ }^{[6]}$ They examined the impact of performance-based teaching methods which included concepts of observation that are task dependent involving lowmental effort, and competency based strategies, which build on what has previously been learned, are task independent, and involve high-mental effort. Teaching methods included lecture, video examples, video assessment, practice sessions, and traditional testing. Peer and individual self-assessment were utilized as well. Findings from the study included that a combination of task orientation and competency based strategies had the best outcomes. The authors also concluded that novice students require some performance-based education strategies prior to initiating CBE strategies. ${ }^{[6]}$

In another study, conducted in Asia, researchers studied 312 undergraduate nursing students in order to examine the impact of CBE on learning outcomes. ${ }^{[3]}$ Half of the students received instruction using CBE strategies (intervention group) and half received traditional instruction methods (control group). The control group received traditional learning methods, which included lectures, case presentations, group discussions, and simulations. The intervention group received CBE methods, including modules that could be completed at the students' pace over the study period. This allowed students to build on previous knowledge and apply that to clinical situations and written reflections to explore and cultivate reasoning and problem solving. All students were assessed following the study period using an objective, structured clinical examination that included communication skills and ethical issues. Students were observed by faculty at each station, where they interacted with standardized patient actors to demonstrate their skills. The results of this study support that students in the intervention group who received CBE teaching methods had significantly higher academic performance than the control group. ${ }^{[3]}$

Experts from the American Council on Education and the Higher Education Policy Program and commissions for higher education have developed five principles in conjunction with several CBE programs across the United States: ${ }^{[8]}$ (1) the degree reflects robust and valid competencies; (2) students are able to learn at a variable pace and are supported in their learning; (3) effective learning resources are available at any time and are reusable; (4) the process for mapping competencies to courses, learning outcomes, and assessments is explicit; and (5) assessments are secure and reliable. Other recommendations include that CBE can be used as a new method of teaching in higher education as long as students are provided the necessary resources and that these are continually updated. ${ }^{[8]}$

\section{METHOD}

\subsection{Context}

In order to use innovative methods to assess clinical skills in an online course, CBEs were added to an online course for psychiatric nurse practitioners. A quality improvement study was conducted to evaluate student knowledge of the content and satisfaction with the method. The setting for this quality improvement study was an online, doctorate-level course for psychiatric mental health nurse practitioners in a large urban university in the Midwest United States. The course is instructed over a 15 -week trimester. The average enrollment in the course is 28 students. This is a distance program that includes students throughout the United States who all have experience as psychiatric nurses, however, the clinical skills being evaluated with the CBE's are at the advanced practice level which they did not have experience in. The focus of the course is mastering the skills of performing and documenting psychiatric assessments in clinical practice. The study sample included 28 students enrolled in the Spring 2017 trimester.

\subsection{Ethical considerations}

An Institutional Review Board exemption at the study site was obtained for this quality improvement project. Identifying data for the participants was not collected. Students were notified via a course announcement that completing the surveys was voluntary and anonymous and would not affect their grades for the course. Identifying information or other demographic data were not collected.

\subsection{Intervention}

Faculty developed four CBE modules to measure student competency in the components in a course that matched objectives for psychiatric assessment: review of systems (ROS), history of present illness (HPI), social history, and mental status exam (MSE). These were posted within the course and in keeping with the principles of CBEs, there was flexibility in the time frame for which they could be completed with the expectation being that half of the CBEs were completed by midterm and all were completed by the end of the term.

Innovative strategies were used to assess student competency

ISSN 1925-4040 E-ISSN 1925-4059 
including students uploading videos of themselves interacting with "mock" patients using GoReact technology which is an online video-recording tool designed to provide feedback for live or user-uploaded videos. ${ }^{[7]}$ Faculty review the video and can type in feedback comments, which automatically pause the video while being entered. When students review the video after it has been assessed, faculty comments are shown on the screen so that students are able to see the comments that pertain to that particular section of the video. Additionally, CBEs were added that included student's assessments of video portrayals of patients with psychiatric symptoms.

Within two weeks of the students' completing the four CBE modules, an administrative assistant sent the modified Student Evaluation of Educational Quality Scale (SEEQ) for each of the modules as well as the Systems Usability Scale (SUS) for the CBE involving the use of GoReact. These were sent via university email accounts and were administered using a college of nursing SurveyMonkey ${ }^{[9]}$ account. The survey was set to obtain anonymous results. SurveyMonkey is used in academic and medical research and provides a simple, convenient electronic method to collect survey data. ${ }^{[9]}$

\subsection{Measures}

To evaluate student knowledge of the content included in the CBEs, knowledge tests were developed by an expert panel of $\mathrm{PhD}$ prepared nursing faculty including one certified as an online educator, and one with expertise in instructional design. The review of systems content was assessed through a 10-item online knowledge test. Questions were developed and then shared within the panel and revisions were made as recommended. To evaluate proficiency in conducting the clinical skill of collecting information for the HPI, a 12-item checklist was developed which included general areas of inquiry to be documented in the HPI Two twelve-item fivepoint Likert scale surveys were used to evaluate the students' responses to the CBE modules and the technology.

The SEEQ scale is a 33-item, 5-point Likert scale instrument available for public use online, developed to measure university students' evaluations ${ }^{[10]}$ and was adapted for use in this study. The response categories range from zero through five or strongly disagree to strongly agree, respectively. The SEEQ is a valid and reliable measure of nine distinct components of teaching effectiveness at the university level, including student ratings and faculty self-evaluation. ${ }^{[10]}$ For this study, the SEEQ was adapted to include only those questions relevant for CBEs in an online course. Twelve questions from the learning, assignment, organization, and examination sections of the SEEQ were included. These modifications were reviewed by an expert panel of $\mathrm{PhD}$ faculty experienced in instructional design and evaluation. The expert panel viewed both the original instrument and the revised instrument to verify that the only items or sections removed were those that were not applicable. For example, an item pertaining to group work was removed since in this course the CBE modules were done on an individual basis.

The SUS was used to evaluate the students' responses to GoReact. The SUS is a widely used tool for digital application assessment. It consists of a 10-item, 5-point Likert scale questionnaire. ${ }^{[11]}$ The scale was developed from a pool of 50 items based on close intercorrelations (+/- 0.7 to 0.9 ) and strong agreement or disagreement, alternating positive with negative items. It has a high level of face validity. ${ }^{[12]}$ The SUS assesses whether users would like to keep using the technology, found it easy to use or cumbersome, would be able to learn to use it quickly, feel a need for technical help using the technology, and their confidence level in using it. $^{[12]}$

In order to evaluate faculty experiences related to the introduction of CBE content into the course, faculty were interviewed to determine what went well and what challenges were encountered as well as any noted improvement in documentation of clinical notes. Although the CBE content was being introduced for the first time in the course, in order to get a general sense of the impact of the content on the student's overall mastery of the content for the course as a whole, scores from the final exam and clinical documentation following the course from the prior year were compared with the year the CBEs were introduced. The results of this QI project were analyzed using descriptive statistics.

\section{Results}

The study was conducted using a one-group, posttest only, quasi-experimental design. ${ }^{[13]}$ Additionally, comparisons were made with overall class performance the prior year. The anonymous surveys obtained from SurveyMonkey were analyzed using descriptive statistics. For the objective assessments, a score of $80 \%$ was required to pass, and for the written and video assessments faculty determined the pass or fail status. To assess the technology and usability of the technology, five surveys were distributed (one SUS and four modified SEEQ) using SurveyMonkey with an average return rate of $56 \%$. Eighteen of the 28 DNP students participated and completed the SUS for a $64 \%$ return rate. As seen in Table 1, results from the SUS on GoReact showed that $89 \%$ agreed that most people would learn to use the technology very quickly, $83 \%$ found it easy to use, and $94 \%$ did not think they needed technical assistance to use it. 
Table 1. Results from system usability scale (SUS) on GoReact

\begin{tabular}{ll}
\hline & Response of strongly agree or agree $(\mathbf{n}=18)$ \\
\hline Most people would learn to use the technology very quickly (\%) & $16(88.89 \%)$ \\
Found technology easy to use (\%) & $15(83.33 \%)$ \\
Did not think technical assistance was needed to use technology (\%) & $17(94.44 \%)$ \\
\hline
\end{tabular}

The survey pertaining to the ROS CBE also had a $64 \%$ return rate on the modified SEEQ. The results seen in Table 2 indicated an overwhelmingly positive response from the students. One hundred percent of the respondents either strongly agreed or agreed that the CBE was intellectually challenging and stimulating. About $89 \%$ indicated that they learned valuable content from the $\mathrm{CBE}$ and that their interest in the subject had increased as a result of the CBE.

Table 2. Results from Student Evaluation of Education Quality (SEEQ) on review of systems (ROS) CBE module

\begin{tabular}{ll}
\hline & Response of strongly agree or agree (n = 18) \\
\hline CBE was intellectually challenging and stimulating (\%) & $18(100 \%)$ \\
Student learned something valuable (\%) & $16(88.89 \%)$ \\
Interest in subject matter increased as a result of the CBE (\%) & $16(88.89 \%)$ \\
\hline
\end{tabular}

Fourteen of the 28 DNP students completed the modified SEEQ survey questions pertaining to the CBE on the concept of social history in nursing for a $50 \%$ return rate. As seen in Table 3, of those who responded $93 \%$ found the CBE intellectually challenging and stimulating, and 86\% reported increased interest in the subject as a result of the CBE. All 14 respondents $(100 \%)$ reported that they learned something valuable.

Table 3. Results from Student Evaluation of Education Quality (SEEQ) on social history CBE module

\begin{tabular}{ll}
\hline & Response of strongly agree or agree $(\mathbf{n}=\mathbf{1 4})$ \\
\hline CBE was intellectually challenging and stimulating (\%) & $13(92.86 \%)$ \\
Student learned something valuable (\%) & $14(100 \%)$ \\
Interest in subject matter increased as a result of the CBE (\%) & $12(85.71 \%)$ \\
\hline
\end{tabular}

Twelve of the 28 DNP students completed the modified SEEQ survey questions pertaining to the MSE CBE in nursing for a $43 \%$ return rate. The results can be seen in Table 4. Of the students who responded, $83 \%$ found the CBE intel- lectually challenging and stimulating, and $92 \%$ reported that they learned something valuable. All 12 respondents (100\%) reported their interest in the subject matter increased as a result of the CBE.

Table 4. Results from Student Evaluation of Education Quality (SEEQ) on mental status exam (MSE) CBE module

\begin{tabular}{ll}
\hline & Response of strongly agree or agree (n = 12) \\
\hline CBE was intellectually challenging and stimulating (\%) & $10(83.33 \%)$ \\
Student learned something valuable (\%) & $11(91.67 \%)$ \\
Interest in subject matter increased as a result of the CBE (\%) & $12(100 \%)$ \\
\hline
\end{tabular}

Sixteen of the 28 DNP students participated and completed the modified SEEQ survey questions pertaining to the CBE on the HPI for a $57 \%$ return rate. Of the 16 who responded, $100 \%$ reported they learned and understood the subject mat- ter in the $\mathrm{CBE}$, and $83 \%$ reported they learned something valuable. Just under $88 \%$ reported their interest in the subject matter increased as a result of the CBE. These results are seen in Table 5. 
Table 5. Results from Student Evaluation of Evaluation Quality (SEEQ) on history of present illness (HPI) CBE module

\begin{tabular}{ll}
\hline & Response of strongly agree or agree (n = 16) \\
\hline Student learned and understood the subject of the CBE (\%) & $16(100 \%)$ \\
Student learned something valuable (\%) & $13(81.25 \%)$ \\
Interest in subject matter increased as a result of the CBE (\%) & $14(87.5 \%)$ \\
\hline
\end{tabular}

Faculty reported that including CBE was beneficial in terms of student success in the course. Although initially creating CBEs can be labor intensive, using the modules ultimately decreased the amount of time spent evaluating the assignments. For example, the CBE assessment modules that were in test format were automatically graded through the learning management system. In addition, the structure of the CBE modules allowed for students to complete them at their own pace and receive feedback along the way. CBE modules are also offered ease in grading versus discussion boards, which are the common method of online teaching.

Faculty reported that it was beneficial that GoReact technology allowed students to record themselves conducting a psychiatric assessment. The faculty could then post comments at specific time points, allowing for more accurate and precise feedback. The end result of these CBE interventions was an improvement in scores on the final standardized interview assignment, a culmination of all CBE modules completed during the course. The overall scores on the standardized interview assessment improved by two points from the prior year. Traditionally, students have had difficulty conducting a successful interview at the end of the course; however, for the trimester in which the CBE modules were included in the course, students earned higher scores on these interviews. For these reasons, faculty involved in the course believe $\mathrm{CBE}$ is an effective teaching method for this content.

In order to further evaluate the use of $\mathrm{CBE}$ modules in this course, course evaluation completed by the students from the prior term the course was last taught were compared with the term during the study period. The mean score in course evaluation from the prior term on effectiveness in how subject matter was presented was 3.18 compared to 3.57 (with 5 as the most effective) when CBE modules were added. In addition, in the student evaluation response to a question about technical difficulties in the prior course, the mean response that students rarely experienced technical difficulties was 3.57 compared to 3.50 (with 5 as the most effective) when GoReact was used. Several faculty observed and scored the standardized patient interviews on campus that the students prepared for using CBEs, and there was agreement that the students performed better this term than in prior terms when CBE modules were not included.

\section{Discussion AND CONCLUSION}

The purpose of this quality improvement study was to incorporate the concept of $\mathrm{CBE}$ in nursing education and to determine the efficacy of this teaching model from a pilot study applying the use of CBE in a doctoral-level nurse practitioner course. The study findings provide preliminary evidence of the effectiveness and impact of CBE modules in an online course to teach psychiatric assessment and documentation in clinical practice. All students in the course were able to successfully complete the CBE modules for course credit.

Results of the study indicated that overall both students and faculty received the CBE very positively. Students found that overall the CBEs were challenging and intellectually stimulating, that the $\mathrm{CBE}$ increased interest in the subject matter, and that they learned the content. Faculty found the initial set up of the CBE modules to be time consuming. However, once they incorporated the modules into the course, they were able to spend less time grading than with traditional instructional methods. Students also performed as well or better than did prior classes on their standardized patient interviews, which the $\mathrm{CBE}$ modules prepared them for.

Overall, based on the positive survey results and faculty feedback, the use of CBE modules in an online DNP nursing course aimed at teaching students psychiatric interviewing and documentation is recommended. CBE also represents a teaching method with emerging evidence in online education. For distance programs that train advanced practice nurses, the use of the video technology GoReact provides a valuable method of assessing students' clinical skills and providing valuable feedback. Per the literature, often a combination of traditional teaching methods along with CBE strategies is beneficial-this parallels the choice of instruction for this online course.

\section{Limitations}

Limitations to the study include that there were different response rates for each of the five surveys, which may have affected the overall results. This likely occurred due to an email glitch that caused the university email system to initially flag some of the surveys as junk mail and place them in quarantine. This was resolved and the students did receive the surveys and reminders to complete them. This could 
have also have contributed to a small sample size which was a further limitation. In addition, the surveys were sent out as a group to all the students to maintain anonymity, which could have contributed to the varying response rates. Sending the surveys individually while maintaining anonymity might improve survey response rates. Another limitation was that this was a pilot study that did not include a control group so that a comparison could not be made between students who were taught the same content using traditional versus CBE methods at the same time. Additionally, a checklist was used to assess the student video of conducting a review of system and for the HPI for this pass-fail assignment, a detailed rubric was not used but was later developed for the course. This lack of a detailed rubric did not impact the students' grade or the faculty from determining a pass-fail designation.

\section{CONFLiCTS OF INTEREST Disclosure}

The authors declare that there is no conflict of interest.

\section{REFERENCES}

[1] U.S. Department of Education. Competency based learning or personalized learning. Available from: https://www.ed.gov/oii-news/competency-based-1 earning-or-personalized-learning.N.d.

[2] Mosalanejad L, Shahsvari S, Sobhanian S, et al. The effect of virtual versus traditional learning in achieving competency-based skills. Turkish Online Journal of Distance Education. 2012; 13(2): 69-75.

[3] Fan J, Wang YH, Chao LF, et al. Performance evaluation of nursing students following competency-based education. Nurse Educ Today. 2015; 35(1): 97-103.

[4] Gravina EW. Competency-based education and its effect on nursing education: A literature review. Teaching and Learning in Nursing. 2016; 12(2): 117-121. https://doi.org/10.1016/j.teln. 201 6.11 .004

[5] Pijl-Zieber EM, Barton S, Konkin J, et al. Competence and competency-based nursing education: Finding our way through the issues. Nurse Educ Today. 2014; 34(5): 676. PMid:24090616 https://doi.org/10.1016/j.nedt.2013.09.007

[6] Fastré GM, van der Klink MR, Amsing-Smit P, et al. Assessment criteria for competency-based education: a study in nursing education. Instructional Science. 2014; 42(6): 971-994. https://doi.org/ $10.1007 / \mathrm{s} 11251-014-9326-5$
[7] GoReact. Features. 2017. Available from: https://get.goreact . com/features/

[8] Johnstone SM, Soares L. Principles for developing competency-based education programs. Change: The Magazine of Higher Learning. 2014; 46(2): 12-19. https://doi.org/10.1080/00091383.201 4.896705

[9] Monkey S. How it works. 2017. Available from: https : //www . su rveymonkey.com/mp/take-a-tour/?ut_source=header

[10] Marsh HW. SEEQ: a reliable valid, and useful instrument for collecting students' evaluations of university teaching. British Journal of Educational Psychology. 1982; 52(1): 77-95. https://doi.org/ $10.1111 / j .2044-8279.1982$.tb02505.x

[11] Brooke J. SUS-A quick and dirty usability scale. Usability Evaluation in Industry. 1996; 189(194): 4-7.

[12] Usability. gov. System usability scale. Available from: https://www.usability.gov/how-to-and-tools/met hods/system-usability-scale.html

[13] Harris AD, McGregor JC, Perencevich EN, et al. The use and interpretation of quasi-experimental studies in medical informatics. J Am MedInform Assoc. 2006; 13(1): 16-23. PMid:16221933 https://doi.org/10.1197/jamia.M1749 